\title{
REVIEW
}

\section{Year in review 2012: Critical Care - respirology}

\author{
Ewan C Goligher ${ }^{1,2}$, Eddy Fan ${ }^{1,2}$ and Arthur S Slutsky ${ }^{1,3^{*}}$
}

\begin{abstract}
Acute respiratory failure is a dominant feature of critical illness. In this review, we discuss 17 studies published last year in Critical Care. The discussion focuses on articles on several topics: respiratory monitoring, acute respiratory distress syndrome, noninvasive ventilation, airway management, secretion management and weaning.
\end{abstract}

\section{Introduction}

This article summarizes 17 studies of the respiratory aspects of critical illness published in Critical Care in 2012. These articles cover a range of issues in the management of respiratory failure, including respiratory monitoring, acute respiratory distress syndrome (ARDS), noninvasive ventilation (NIV), airway management, secretion management, and weaning from mechanical ventilation.

\section{Respiratory monitoring}

Approaches to setting the optimum positive endexpiratory pressure (PEEP) level have been studied for at least the past four decades, and yet there is still no widely accepted method. Breen and Mazumdar [1] suggested that capnography could be employed to monitor the effects of PEEP on both pulmonary and cardiac function. The slope of phase III of the capnogram reflects the extent of heterogeneity in carbon dioxide $\left(\mathrm{CO}_{2}\right)$ tensions across lung units of varying time constants [2]. Consequently, any lung pathology that increases the heterogeneity of time constants and alveolar $\mathrm{CO}_{2}$ tensions will increase the phase III slope, which may be monitored to assess the effects of PEEP on ventilation. The physiological meaning of the phase III slope was recently shown to depend on respiratory system mechanics [3]. PEEP-induced changes in

\footnotetext{
* Correspondence: slutskya@smh.ca

${ }^{1}$ Interdepartmental Division of Critical Care Medicine, University of Toronto, Toronto M5B1W8, Canada

${ }^{3}$ Keenan Research Center at the Li Ka Shing Knowledge Institute of St Michael's Hospital, 209 Victoria Street, Toronto, Ontario M5B1W8, Canada Full list of author information is available at the end of the article
}

phase III slope were strongly related to airway resistance in patients with low elastance. By contrast, in patients with high elastance, changes in phase III slope largely reflected changes in elastance. Of note, time domain and volumetric capnography revealed similar changes in the phase III slope, supporting the validity of monitoring capnography in the time domain as is typical in clinical practice. Capnography may be useful to monitor the effects of PEEP at the bedside; however, the precise physiological significance of the phase III slope depends on respiratory elastance.

$\mathrm{CO}_{2}$ clearance and alveolar dead space are determined by several physiological factors: venous return, venous $\mathrm{CO}_{2}$ content, distribution of ventilation, and the extent of respiratory gas mixing within the alveoli and respiratory bronchioles during each breath [1,4]. Gas mixing is enhanced both by the abrupt cessation of high endinspiratory flows (achieved by an accelerating flow profile) and by increasing the mean distribution time (achieved by a post-inspiratory pause). However, these maneuvers negatively interact because an accelerating flow profile tends to reduce mean distribution time, as most of tidal volume is delivered late in the breath, reducing the residence time in the alveoli. When the postinspiratory pause was kept short $(<10 \%$ of cycle time), increasing mean distribution time by employing a decelerating flow profile enhanced gas mixing and $\mathrm{CO}_{2}$ elimination despite the resulting fall in end-inspiratory flow [5]. By contrast, an accelerating flow profile resulted in greater $\mathrm{CO}_{2}$ elimination than a decelerating flow profile when the post-inspiratory pause was prolonged (35\% of cycle time). $\mathrm{CO}_{2}$ elimination was enhanced by up to $20 \%$ following these maneuvers. Given that lung protection with low tidal volume ventilation is frequently hampered by severe respiratory acidosis, clinicians may find it useful to try these maneuvers to maintain tidal volumes at $6 \mathrm{ml} / \mathrm{kg}$, or to lower tidal volumes even further in an effort to reduce ventilator-induced lung injury (VILI), although caution is warranted given the theoretical deleterious effects of accelerating inspiratory flow on VILI. 


\section{Acute respiratory distress syndrome Mechanisms of lung injury}

Interstitial edema is a prominent feature of ARDS. Several pathophysiologic mechanisms for the presence of alveolar edema have been identified, including derangements in chloride flux (the movement of chloride ions from the alveolar to interstitial spaces), an important determinant of fluid clearance [6]. Chloride flux is mediated by a series of membrane channels including the $\gamma$-amino-butyric acid (GABA)-sensitive chloride channel. Chintagari and Liu [7] examined the effects of stimulating GABA receptors on chloride flux and VILI. GABA stimulation reduced edema and mitigated a number of histopathologic features of VILI, leading the authors to speculate that GABA receptor stimulation may exert a protective effect against VILI.

The systemic inflammatory effects of acute lung injury were established well over a decade ago [8]. 'Biotrauma' as this phenomenon has been termed - provides the mechanistic basis for the mortality benefit of lungprotective ventilation demonstrated in the ARMA trial [9]. Nevertheless, it is possible that hypoxemia or circulatory shock in some patients with ARDS, rather than systemic spread of intrapulmonary inflammation per se, might contribute to multiorgan dysfunction. Heuer and colleagues examined the causes of multiorgan injury in a porcine acid aspiration model [10]. Within 4 hours of intrabronchial instillation of hydrochloric acid, they found inflammatory infiltrates and/or tissue necrosis within the heart (myocardium and conduction system), kidneys (predominantly in the tubules of the renal cortex), liver and brain (hippocampal regions); no inflammation was identified in control animals. Although acid instillation widened the alveolar-arterial gradient, the partial pressure of oxygen $\left(\mathrm{P}_{\mathrm{a}} \mathrm{O}_{2}\right)$ never fell below $250 \mathrm{mmHg}$ in either study group (acid instillation versus control) during the entire experiment and cardiac output was stable and similar in both study groups. The authors concluded that hypoxemia and hemodynamic instability could not account for the multiorgan inflammation observed following acid aspiration. These observations reinforce the theory that pulmonary inflammation, associated with the loss of pulmonary compartmentalization [11], contributes to the development of multiorgan dysfunction following the onset of acute lung injury.

\section{Diagnosis}

Given the rarity of pulmonary capillary wedge pressure monitoring in contemporary practice [12], acute lung injury is generally differentiated from heart failure on clinical grounds; accurate determination of the contribution of cardiac dysfunction to lung edema can be challenging. Kushimoto and colleagues [13] tested the utility of PiCCO-based measurements of extravascular lung water index (EVLWI) and pulmonary vascular permeability index (PVPI) in establishing the diagnosis of ARDS in a heterogeneous clinical population of ARDS patients (78\%), heart failure (10\%), or pleural effusion (12\%). PVPI was higher in ARDS patients than non-ARDS patients and reliably discriminated between ARDS and non-ARDS (area under the receiver operating characteristic curve $=0.89$ ). Unfortunately, because the experts who established the gold standard diagnosis were not blinded to EVLWI values, this discriminant function may be considerably inflated. PVPI and EVLWI are mathematically coupled; consequently, patients with higher PVPI tend to have higher EVLWI values. The EVLWI values may have influenced the reviewers' decision to classify patients. Further work is required to establish the true validity of EVLWI and PVPI for the diagnosis of ARDS.

\section{Lung recruitment}

Higher PEEP may be beneficial in severe ARDS [14], but there is considerable disagreement over how, when and in whom to apply higher PEEP levels safely (that is, recruit without overdistending healthy lung). de Matos and colleagues [15] reported the outcomes of a cohort of patients with moderate and severe ARDS $(n=51)$ subjected to a 'maximal recruitment strategy' [16]. Employing an aggressive recruitment strategy with a maximum airway pressure of $60 \mathrm{cmH}_{2} \mathrm{O}$, they were able to recruit substantial amounts of previously collapsed non-aerated lung (median 45\%). PEEP was maintained above the closing pressure (mean $25 \pm 3 \mathrm{cmH}_{2} \mathrm{O}$ ) for 48 hours and titrated down gradually thereafter to maintain a ratio of partial pressure of oxygen to fraction of inspired oxygen $\left(\mathrm{P}_{\mathrm{a}} \mathrm{O}_{2}: \mathrm{F}_{\mathrm{i}} \mathrm{O}_{2}\right)$ greater than $300 \mathrm{mmHg}$. This protocol was able to maintain a $\mathrm{P}_{\mathrm{a}} \mathrm{O}_{2}: \mathrm{F}_{\mathrm{i}} \mathrm{O}_{2}>300 \mathrm{mmHg}$ in almost all patients over the following week. Hospital mortality for this cohort was 33\%. The authors concluded that applying very high PEEP was safe and effectively recruited injured lung. The study protocol achieved higher rates of lung recruitment than observed previously [17], perhaps because higher airway pressures were applied or because of differences between the patient populations (early ARDS). These data suggest that dramatic alveolar recruitment can be achieved and maintained in many cases. It remains unclear whether the putative benefits of this aggressive ventilatory strategy outweigh the risks of alveolar overdistention and hemodynamic compromise.

Ideally, PEEP is titrated to prevent cyclic alveolar recruitment and collapse while minimizing overdistention in the open lung [18]. The 'optimal PEEP' that achieves these goals may vary considerably between patients. Techniques to identify optimal PEEP in individual patients lack either validity or feasibility. The forced oscillation technique (FOT) is an interesting approach to monitoring changes in respiratory mechanics. A loudspeaker is attached to the 
ventilator circuit to induce rapid $(5 \mathrm{~Hz})$ and very small fluctuations in airway pressure $\left(2 \mathrm{cmH}_{2} \mathrm{O}\right)$. Measurement of resulting oscillations in flow and pressure permit computation of respiratory reactance (a measure of the elastic properties of the lung and chest wall). In ARDS, the respiratory reactance measured by FOT is strongly related to the proportion of collapsed tissue within the lung [19]. Because measurements are derived from oscillations applied at a constant mean airway pressure, FOT can also be employed to monitor changes in elastance between endexpiration and end-inspiration (a measure of tidal stress). During a decremental PEEP trial, both the tidal change in elastance and the end-expiratory elastance were minimized at similar intermediate values of PEEP, suggesting that both lung collapse and tidal strain were minimized at these 'optimal PEEP' levels [20]. These findings raise the possibility that monitoring forced oscillations might provide a feasible means of identifying the PEEP level at which both alveolar collapse and overdistention are minimized.

Hartmann and colleagues [21] compared the effect of increases in respiratory rate to increases in PEEP to minimize tidal atelectasis. Cyclic alveolar recruitment was monitored by respiratory oscillations in $\mathrm{P}_{\mathrm{a}} \mathrm{O}_{2}$. Increasing the respiratory frequency from 5 to 15 breaths per minute reduced respiratory $\mathrm{P}_{\mathrm{a}} \mathrm{O}_{2}$ oscillations but could not maintain a stable $\mathrm{P}_{\mathrm{a}} \mathrm{O}_{2}$ thereafter. Increasing the PEEP reduced respiratory $\mathrm{P}_{\mathrm{a}} \mathrm{O}_{2}$ oscillations and maintained stable $\mathrm{P}_{a} \mathrm{O}_{2}$ values. These findings suggest that increasing PEEP is a more reliable means of maintaining alveolar recruitment than relatively small changes in respiratory frequency.

Transpulmonary pressure, rather than airway pressure, is the central determinant of lung volume and strain for a given lung compliance. While airway pressure is commonly used as a surrogate for transpulmonary pressure, the relationship between two pressures is frequently confounded by conditions that either (a) alter chest wall compliance or (b) shift the transpulmonary pressurevolume curve to the right (or both). Intra-abdominal hypertension is a useful example. High intra-abdominal pressure forces the diaphragm upward and transmits positive pressure into the thoracic cavity. The resulting fall in mean transpulmonary pressure is associated with lower lung volumes and contributes to atelectasis and hypoxemia, particularly in the setting of concomitant lung injury. Regli and colleagues [22] studied the effects of high PEEP in a porcine model of acute lung injury with varying levels of intra-abdominal hypertension. Increasing intra-abdominal pressure increased both lung elastance and chest wall elastance. Conversely, an increased gradient between PEEP and intra-abdominal pressure was strongly associated with higher endexpiratory lung volumes $\left(R^{2}=0.59, \quad P<0.001\right)$ and improved oxygenation $\left(R^{2}=0.52, P<0.001\right)$. The cardiac output fell by approximately $30 \%$ at the highest level of intra-abdominal pressure and matching PEEP. In contrast to previous work [23] where lower abdominal pressures were studied, these findings suggest that matching PEEP to intra-abdominal pressure can dramatically recruit the lung, and this may prevent VILI. Caution regarding hemodynamic effects in individual patients is strongly warranted.

In the early days of mechanical ventilation, negative pressure ventilation was the dominant means of generating transpulmonary pressure. Although rare in contemporary practice, negative pressure ventilation has a number of potential advantages over positive pressure ventilation: better oxygenation (largely due to improved distribution of ventilation) and potentially reduced VILI compared to positive pressure ventilation [24,25]. However, the observed salutary effects of negative pressure ventilation may arise from differences in pressure-time profile and lung volume history rather than altered distribution of transpulmonary pressure [26]. When negative pressure ventilation was employed in humans with ARDS by a 'tank respirator' (that is, iron lung) surrounding the whole body below the neck, oxygenation was maintained or improved at lower transpulmonary pressures [27]. Despite a fall in arterial pressure and central venous pressure, intrathoracic blood volume and cardiac output increased. These findings highlight the importance of the distribution of ventilation: negative pressure ventilation (including negative pleural pressure achieved by respiratory muscle activity during spontaneous breathing) may achieve a more optimal, less injurious distribution of ventilation [28]. However, whether this translates into improvements in clinically important outcomes likely depends critically on finding the optimal level of negative inspiratory pressure as excessive inspiratory effort or negative pressure may generate injurious transpulmonary pressures and exacerbate VILI.

\section{General respiratory care}

\section{Noninvasive ventilation}

NIV is clearly beneficial for a number of causes of acute respiratory failure, such as acute exacerbations of chronic obstructive pulmonary disease and acute cardiogenic pulmonary edema [29]. Immunosuppressed patients, including patients with hematological malignancy, are at higher risk of nosocomial infections and NIV may be of particular benefit in this population. Two small randomized trials of NIV in immunosuppressed patients suggested that NIV may prevent intubation and reduce mortality [30,31]. Molina and colleagues [32] described clinical outcomes with NIV in a large cohort of patients $(n=300)$ with a broad range of hematologic malignancies who were admitted to the ICU with acute 
respiratory failure. Compared to initial invasive ventilation, NIV was associated with lower adjusted mortality. However, NIV failure was associated with higher mortality than initial invasive ventilation. Despite multivariable adjustment, these findings are probably biased due to residual confounding by indication and by underlying disease severity (since NIV success or failure will reflect disease severity). However, given the possible harm associated with NIV failure, NIV should be applied judiciously in these patients - careful patient selection and monitoring is essential. Further randomized trials of NIV in this extremely ill patient population are warranted.

\section{Airway management}

Endotracheal intubation is often challenging in critically ill patients [33]. The C-MAC ${ }^{\circ}$ video laryngoscope (Karl Storz GmbH \& Co. KG, Tuttlingen, Germany), one of many adjunct devices available for difficult intubations, employs a blade fashioned after the standard Macintosh laryngoscope blade combined with a camera placed near the tip of the blade connected to a peripheral video screen. The outcome following the routine use of the CMAC by anesthesiologists was recently evaluated in a single ICU center [34]. Although the difficult intubation rate (two or more failed intubation attempts) was not lower in the overall study population after instituting routine use of the C-MAC laryngoscope (7\% versus 3\%, $P$-value not reported), patients predicted to be difficult to intubate were intubated on the first attempt more frequently (79\% versus 56\% for C-MAC and manual laryngoscopy, respectively, $P=0.03)$. The glottis was more easily visualized with the C-MAC system and physiciansin-training performed intubation more frequently $(59 \%$ versus $36 \%, P<0.001$ ) after routine C-MAC deployment. Routine use of the C-MAC system is safe and feasible and may enhance trainee education in airway management.

Although bedside percutaneous tracheostomy is now a common procedure in critically ill patients, anatomical variations in airway or neck anatomy occasionally make this approach challenging. Ultrasound guidance has been shown to reduce the complications of a number of common ICU procedures and could aid percutaneous tracheotomy. Recently, ultrasound guidance was employed during percutaneous tracheostomy in 50 consecutive ICU patients, 52\% of whom were obese [35]. Ultrasoundguided tracheostomy was successfully performed at the bedside in all 50 patients and the complication rate did not differ between obese and non-obese patients. The puncture site (initially selected by palpation) was changed after ultrasound examination in $50 \%$ of cases due to the position of the thyroid, presence of aberrant vessels, or tracheal deviation. Ultrasound guidance therefore seems feasible and may enhance the safety of bedside percutaneous tracheostomy.

\section{Secretion management}

Pulmonary secretions frequently impair liberation from mechanical ventilation and often contribute to postextubation respiratory failure. Impairment in the cough mechanism due to endotracheal intubation, muscular weakness, or sedation contributes to mucous plugging and respiratory failure. Various physical maneuvers can be employed to mimic and enhance cough effectiveness. Manual hyperinflation involves slow manual inspiration by a ventilation bag (to a volume around $50 \%$ greater than usual tidal volume) followed by an inspiratory pause and rapid expiration with high expiratory flow (by rapidly releasing the pressure on the ventilation bag). Theoretically this maneuver should propel secretions from small airways toward larger central airways. A recent systematic review found that sputum clearance was not consistently improved by manual hyperinflation [36]. Moreover, ICU and hospital length of stay were not different in heterogeneous patient populations (including brain-injured patients) who were treated with or without this approach.

Mechanical insufflation-exsufflation (MIE) is a similar technique employing a Cough Assist $^{\bullet}$ device (Philips Respironics, Carlsbad, CA, USA) to apply cyclic positive and negative pressures $\left( \pm 40 \mathrm{cmH}_{2} \mathrm{O}\right)$ to the airway via endotracheal tube or oronasal mask. Negative airway pressures may be accompanied by an abdominal thrust to enhance expiratory flow. Gonçalves and colleagues [37] hypothesized that MIE might reduce the rate of post-extubation respiratory failure. They randomized a heterogeneous cohort of mechanically ventilated patients to standard care $(n=40)$ or MIE immediately prior to and three times daily for 48 hours following extubation $(\mathrm{n}=35)$. The reintubation rate was lower in the patients assigned to MIE (17\% versus $48 \%, P<0.05$ ). However, the initial duration of mechanical ventilation (MV) was shorter in MIE patients, indicating an important imbalance between groups that may have confounded the difference in outcome. ICU length of stay was not significantly different between the two groups.

When applied consistently to appropriate patient populations, these maneuvers to mimic cough may enhance secretion clearance and improve clinical outcomes. However, well-designed large randomized clinical trials of these methods are lacking. Based on the encouraging findings of the above studies, these maneuvers merit further investigation.

\section{Weaning}

'Closed loop' ventilation employs automated algorithmbased titration of ventilator settings based on various respiratory parameters monitored by the ventilator. By responding more rapidly and consistently to changes in patient respiratory status and removing the need for 
human intervention, 'closed loop' ventilation may accelerate liberation from mechanical ventilation. Data supporting clinical benefit are lacking and a randomized trial of closed loop control of pressure support ventilation failed to show a reduction in duration of ventilation [38]. Nevertheless, this area of research is in its infancy, and most reports focus on establishing the feasibility and safety of closed loop systems in various populations. Adaptive support ventilation (ASV) is a 'semi-automatic' mode that adjusts driving pressure to achieve a set minute ventilation by some optimal combination of tidal volume and respiratory rate determined based on the time constant of the respiratory system [39]. Fully automatic 'closed loop' ASV automatically adjusts the target minute ventilation, PEEP and $\mathrm{F}_{\mathrm{i}} \mathrm{O}_{2}$ based on the end-tidal $\mathrm{CO}_{2}$ and peripheral oxygen saturation. In a pediatric population comparing closed loop ASV to standard ASV and pressure support ventilation within patients, closed loop ASV obtained similar values for respiratory rate, tidal volume, peak airway pressure, and minute ventilation (although tidal volume variability was higher) [40]. Ventilator settings were adjusted more frequently during closed loop control. Future evaluations of the utility of closed loop modes for weaning children safely and more quickly from mechanical ventilation will be of interest.

Sedation often poses a major obstacle to weaning from mechanical ventilation [41]. Randomized trials of various sedation titration strategies suggest that sedation protocols accelerate weaning $[42,43]$ but adherence to established protocols remains inconsistent. Amaral and colleagues [44] measured the impact of a multi-faceted quality improvement intervention aimed at enhancing adherence to sedation protocols. Despite reasonably high levels of protocol adherence at baseline (approximately $80 \%)$, the intervention nevertheless significantly improved adherence (to 96\%). This improvement was associated with a significant reduction in the length of mechanical ventilation (fitted median 14.5\%, interquartile range 13.8 to $15.8 \%$ ) and unit-level ventilator-days per year (reduction of 503 days, 95\% confidence interval 301 to 729 days). These data suggest that the effort required to attain very high levels of sedative protocol compliance is highly worthwhile, a finding that may be applicable to a wide range of quality control problems in critical care (tidal volume limitation, venous thromboembolism prophylaxis, early mobilization, and so on).

\section{Conclusion}

The management of respiratory failure is the "bread and butter' of critical care medicine. Nevertheless, much uncertainty exists regarding optimal management of many respiratory problems. The studies published in 2012 provide many important insights into these problems and provide an invaluable contribution to the continued pursuit of clinical and scientific excellence in the ICU.

\section{Abbreviations}

ARDS: Acute respiratory distress syndrome; ASV: Adaptive support ventilation; $\mathrm{CO}_{2}$ : Carbon dioxide; EVLWI: Extravascular lung water index; $\mathrm{F}_{\mathrm{i}} \mathrm{O}_{2}$ : Fraction of inspired oxygen; FOT: Forced oscillation technique; GABA: $y$-amino-butyric acid; MIE: Mechanical insufflation-exsufflation; NIV: Noninvasive ventilation; $\mathrm{P}_{\mathrm{a}} \mathrm{O}_{2}$ : Partial pressure of oxygen; PEEP: Positive end-expiratory pressure; PVPI: Pulmonary vascular permeability index; VILI: Ventilator-induced lung injury.

\section{Competing interests}

The authors declare that they have no competing interests.

\section{Author details}

${ }^{1}$ Interdepartmental Division of Critical Care Medicine, University of Toronto, Toronto M5B1W8, Canada. ${ }^{2}$ Department of Medicine, University Health Network, Toronto M5G 2C4, Canada. ${ }^{3}$ Keenan Research Center at the Li Ka Shing Knowledge Institute of St Michael's Hospital, 209 Victoria Street, Toronto, Ontario M5B1W8, Canada.

\section{Published: 22 Nov 2013}

\section{References}

1. Breen $\mathrm{PH}$, Mazumdar B: How does positive end-expiratory pressure decrease CO2 elimination from the lung? Respir Physiol 1996, 103:233-242.

2. Tusman G, Areta M, Climente C, Plit R, Suarez-Sipmann F, Rodríguez-Nieto MJ, Peces-Barba G, Turchetto E, Bohm SH: Effect of pulmonary perfusion on the slopes of single-breath test of CO2. J Appl Physiol 2005, 99:650-655.

3. Babik B, Csorba Z, Czövek D, Mayr PN, Bogáts G, Peták F: Effects of respiratory mechanics on the capnogram phases: importance of dynamic compliance of the respiratory system. Crit Care 2012, 16:R177.

4. Devaquet J, Jonson B, Niklason L, Si Larbi A-G, Uttman L, Aboab J, Brochard $L$ : Effects of inspiratory pause on $\mathrm{CO} 2$ elimination and arterial $\mathrm{PCO} 2$ in acute lung injury. J Appl Physiol 2008, 105:1944-1949.

5. Aboab J, Niklason L, Uttman L, Brochard L, Jonson B: Dead space and $\mathrm{CO}_{2}$ elimination related to pattern of inspiratory gas delivery in ARDS patients. Crit Care 2012, 16:R39.

6. Mutlu GM, Sznajder J: Mechanisms of pulmonary edema clearance. Am J Physiol Lung Cell Mol Physiol 2005, 289:L685-L695.

7. Chintagari NR, Liu L: GABA receptor ameliorates ventilator-induced lung injury in rats by improving alveolar fluid clearance. Crit Care 2012, 16:R55.

8. Tremblay L, Valenza F, Ribeiro SP, Li J, Slutsky AS: Injurious ventilatory strategies increase cytokines and c-fos m-RNA expression in an isolated rat lung model. J Clin Invest 1997, 99:944-952.

9. The Acute Respiratory Distress Syndrome Network: Ventilation with lower tidal volumes as compared with traditional tidal volumes for acute lung injury and the acute respiratory distress syndrome. N Engl J Med 2000, 342:1301-1308.

10. Heuer JF, Sauter P, Pelosi P, Herrmann P, Brück W, Perske C, Schöndube F, Crozier TA, Bleckmann A, Beißbarth T, Quintel M: Effects of pulmonary acid aspiration on the lungs and extra-pulmonary organs: a randomized study in pigs. Crit Care 2012, 16:R35.

11. Slutsky AS: Lung injury caused by mechanical ventilation. Chest 1999 , 116:9S-15S.

12. Rubenfeld GD, McNamara-Aslin E, Rubinson L: The pulmonary artery catheter, 1967-2007: rest in peace? JAMA 2007, 298:458-461.

13. Kushimoto S, Taira Y, Kitazawa Y, Okuchi K, Sakamoto T, Ishikura H, Endo T, Yamanouchi S, Tagami T, Yamaguchi J, Yoshikawa K, Sugita M, Kase Y, Kanemura T, Takahashi H, Kuroki Y, Izumino H, Rinka H, Seo R, Takatori M, Kaneko T, Nakamura T, Irahara T, Saito N, Watanabe A: The PiCCO Pulmonary Edema Study Group: The clinical usefulness of extravascular lung water and pulmonary vascular permeability index to diagnose and characterize pulmonary edema: a prospective multicenter study on the quantitative differential diagnostic definition for acute lung injury/acute respiratory distress syndrome. Crit Care 2012, 16:R232.

14. Briel M, Meade M, Mercat A, Brower RG, Talmor D, Walter SD, Slutsky AS, Pullenayegum E, Zhou Q, Cook D, Brochard L, Richard J-CM, Lamontagne F, 
Bhatnagar N, Stewart TE, Guyatt G: Higher vs lower positive end-expiratory pressure in patients with acute lung injury and acute respiratory distress syndrome: systematic review and meta-analysis. JAMA 2010, 303:865-873.

15. de Matos GFJ, Stanzani F, Passos RH, Fontana MF, Albaladejo R, Caserta RE, Santos DCB, Borges JB, Amato MBP, Barbas CSV: How large is the lung recruitability in early acute respiratory distress syndrome: a prospective case series of patients monitored by computed tomography. Crit Care 2012, 16:R4.

16. Borges JB, Okamoto VN, Matos GFJ, Caramez MPR, Arantes PR, Barros F, Souza CE, Victorino JA, Kacmarek RM, Barbas CSV, Carvalho CRR, Amato MBP: Reversibility of lung collapse and hypoxemia in early acute respiratory distress syndrome. Am J Respir Crit Care Med 2006, 174:268-278.

17. Gattinoni L, Caironi P, Cressoni M, Chiumello D, Ranieri VM, Quintel M, Russo S, Patroniti N, Cornejo R, Bugedo G: Lung recruitment in patients with the acute respiratory distress syndrome. N Engl J Med 2006, 354:1775-1786.

18. Brochard L: New goals for positive end-expiratory pressure in acute respiratory distress syndrome: a paradigm shift or the end of an area of uncertainty? Am J Respir Crit Care Med 2010, 181:528-530.

19. Dellacà RL, Zannin E, Kostic P, Olerud MA, Pompilio PP, Hedenstierna G, Pedotti A, Frykholm P: Optimisation of positive end-expiratory pressure by forced oscillation technique in a lavage model of acute lung injury. Intensive Care Med 2011, 37:1021-1030.

20. Zannin E, Dellacà RL, Kostic P, Pompilio PP, Larsson A, Pedotti A, Hedenstierna G, Frykholm P: Optimizing positive end-expiratory pressure by oscillatory mechanics minimizes tidal recruitment and distension: an experimental study in a lavage model of lung injury. Crit Care 2012, 16:R217.

21. Hartmann EK, Boehme S, Bentley A, Duenges B, Klein KU, Elsaesser A, Baumgardner JE, David M, Markstaller $K$ : Influence of respiratory rate and end-expiratory pressure variation on cyclic alveolar recruitment in an experimental lung injury model. Crit Care 2012, 16:R8.

22. Regli A, Mahendran R, Fysh ET, Roberts B, Noffsinger B, De Keulenaer BL, Singh $B$, van Heerden PV: Matching positive end-expiratory pressure to intra-abdominal pressure improves oxygenation in a porcine sick lung model of intra-abdominal hypertension. Crit Care 2012, 16:R208.

23. Krebs J, Pelosi P, Tsagogiorgas C, Alb M, Luecke T: Effects of positive end-expiratory pressure on respiratory function and hemodynamics in patients with acute respiratory failure with and without intra-abdominal hypertension: a pilot study. Crit Care 2009, 13:R160.

24. Chierichetti M, Engelberts D, El-Khuffash A, Babyn P, Post M, Kavanagh BP: Continuous negative abdominal distension augments recruitment of atelectatic lung. Crit Care Med 2012, 40:1864-1872.

25. Grasso F, Engelberts D, Helm E, Frndova H, Jarvis S, Talakoub O, McKerlie C, Babyn P, Post M, Kavanagh BP: Negative-pressure ventilation: better oxygenation and less lung injury. Am J Respir Crit Care Med 2008, 177:412-418.

26. Engelberts D, Malhotra A, Butler JP, Topulos GP, Loring SH, Kavanagh BP: Relative effects of negative versus positive pressure ventilation depend on applied conditions. Intensive Care Med 2012, 38:879-885.

27. Raymondos K, Molitoris U, Capewell M, Sander B, Dieck T, Ahrens J, Weilbach C, Knitsch W, Corrado A: Negative- versus positive-pressure ventilation in intubated patients with acute respiratory distress syndrome. Crit Care 2012, 16:R37.

28. Mauri T, Bellani G, Confalonieri A, Tagliabue P, Turella M, Coppadoro A, Citerio G, Patroniti N, Pesenti A: Topographic distribution of tidal ventilation in acute respiratory distress syndrome: effects of positive end-expiratory pressure and pressure support. Crit Care Med 2013, 41:1664-1673.

29. Keenan SP, Sinuff T, Burns KEA, Muscedere J, Kutsogiannis J, Mehta S, Cook DJ, Ayas N, Adhikari NKJ, Hand L, Scales DC, Pagnotta R, Lazosky L, Rocker G, Dial S, Laupland K, Sanders K, Dodek P: Canadian Critical Care Trials Group/Canadian Critical Care Society Noninvasive Ventilation Guidelines Group: Clinical practice guidelines for the use of noninvasive positive-pressure ventilation and noninvasive continuous positive airway pressure in the acute care setting. CMAJ 2011, 183:E195-E214.

30. Antonelli M, Conti G, Bufi M, Costa MG, Lappa A, Rocco M, Gasparetto A, Meduri GU: Noninvasive ventilation for treatment of acute respiratory failure in patients undergoing solid organ transplantation: a randomized trial. JAMA 2000, 283:235-241.
31. Hilbert G, Gruson D, Vargas F, Valentino R, Gbikpi-Benissan G, Dupon M, Reiffers J, Cardinaud JP: Noninvasive ventilation in immunosuppressed patients with pulmonary infiltrates, fever, and acute respiratory failure. $N$ Engl J Med 2001, 344:481-487.

32. the EMEHU study investigators, Molina R, Bernal T, Borges M, Zaragoza R, Bonastre J, Granada RM, Rodriguez-Borregán JC, Núñez K, Seijas I, Ayestarán I, Albaiceta GM: Ventilatory support in critically ill hematology patients with respiratory failure. Crit Care 2012, 16:R133.

33. De Jong A, Molinari N, Terzi N, Mongardon N, Arnal J-M, Guitton C, Allaouchiche B, Paugam-Burtz C, Constantin J-M, Lefrant J-Y, Leone M, Papazian L, Asehnoune K, Maziers N, Azoulay E, Pradel G, Jung B, Jaber S: AzuRéa Network for the Frida-Réa Study Group: Early identification of patients at risk for difficult intubation in the intensive care unit. Am J Respir Crit Care Med 2013, 187:832-839.

34. Noppens RR, Geimer S, Eisel N, David M, Piepho T: Endotracheal intubation using the ${\mathrm{C}-M A C^{\oplus}}^{\oplus}$ video laryngoscope or the Macintosh laryngoscope: a prospective, comparative study in the ICU. Crit Care 2012, 16:R103.

35. Guinot P-G, Zogheib E, Petiot S, Marienne J-P, Guerin A-M, Monet P, Zaatar $\mathrm{R}$, Dupont $\mathrm{H}$ : Ultrasound-guided percutaneous tracheostomy in critically ill obese patients. Crit Care 2012, 16:R40.

36. Paulus F, Binnekade JM, Vroom MB, Schultz MJ: Benefits and risks of manual hyperinflation in intubated and mechanically ventilated intensive care unit patients: a systematic review. Crit Care 2012, 16:R145.

37. Gonçalves MR, Honrado T, Winck JC, Paiva JA: Effects of mechanical insufflation-exsufflation in preventing respiratory failure after extubation: a randomized controlled trial. Crit Care 2012, 16:R48.

38. Schädler D, Engel C, Elke G, Pulletz S, Haake N, Frerichs I, Zick G, Scholz J, Weiler N: Automatic control of pressure support for ventilator weaning in surgical intensive care patients. Am J Respir Crit Care Med 2012, 185:637-644.

39. Burns KEA, Lellouche F, Lessard MR: Automating the weaning process with advanced closed-loop systems. Intensive Care Med 2008, 34:1757-1765.

40. Jouvet P, Eddington A, Payen V, Bordessoule A, Emeriaud G, Gasco RL, Wysocki M: A pilot prospective study on closed loop controlled ventilation and oxygenation in ventilated children during the weaning phase. Crit Care 2012, 16:R85.

41. Patel SB, Kress JP: Sedation and analgesia in the mechanically ventilated patient. Am J Respir Crit Care Med 2012, 185:486-497.

42. Kress JP, Pohlman AS, O'Connor MF, Hall JB: Daily interruption of sedative infusions in critically ill patients undergoing mechanical ventilation. N Engl J Med 2000, 342:1471-1477.

43. Strøm T, Martinussen T, Toft P: A protocol of no sedation for critically ill patients receiving mechanical ventilation: a randomised trial. Lancet 2010, 375:475-480.

44. Amaral AC, Kure $L$, Jeffs $A$ : Effects of increasing compliance with minimal sedation on duration of mechanical ventilation: a quality improvement intervention. Crit Care 2012, 16:R78.

\section{$10.1186 / \mathrm{cc} 13129$}

Cite this article as: Goligher et al: Year in review 2012: Critical Care respirology. Critical Care 2013, 17:249 